\title{
Epidemiology of severe trauma patients treated by plastic surgeons: A 7-year study at a single regional trauma center in South Korea
}

\author{
Joo Sung Jung, Dong Hee Kang, Nam Kyu Lim \\ Department of Plastic and Reconstructive Surgery, Dankook University Hospital, Cheonan, Korea
}

Background After the laws regulating emergency medicine were amended in 2012, regional trauma centers were established in South Korea. Plastic surgeons specialize in the simultaneous surgical care of patients with facial trauma, burns, and complicated wounds. The objective of this study was to evaluate the role of the plastic surgery department in treating severe trauma patients.

Methods From January 2012 to December 2018, we enrolled 366 severe trauma patients with an Injury Severity Score (ISS) over 15 who received treatment by specialists in the plastic surgery department. Of these patients, 298 (81.4\%) were male, and their mean age was 51.35 years (range, 6-91 years). The average ISS was 22.01 points (range, 16-75 points).

Results The most common diagnosis was facial trauma (95.1\%), and facial bone fracture $(65.9 \%)$ was most common injury within this subgroup. Patients were referred to 1.8 departments on average, with the neurosurgery department accounting for a high proportion of collaborations (37.0\%). The most common cause of trauma was traffic accidents (62.3\%), and the average length of stay in the general ward and intensive care unit was 36.90 and 8.01 days, respectively. Most patients were discharged home (62.0\%) without additional transfer or readmission.

Conclusions Through this study, we scoped out the role of the specialty of plastic surgery in the multidisciplinary team at regional trauma centers. These results may have implications for trauma system planning.

Keywords Trauma centers / Surgery, plastic / Traumatology / Epidemiology

\author{
Correspondence: Nam Kyu Lim \\ Department of Plastic and \\ Reconstructive Surgery, Dankook \\ University Hospital, 201 Manghyang- \\ ro, Dongnam-gu, Cheonan 31116, \\ Korea \\ Tel: +82-41-550-6477 \\ Fax: +82-41-554-6477 \\ E-mail: linakyer@dkuh.co.kr; \\ linakyer@daum.net
}

\section{INTRODUCTION}

The laws regulating emergency medical services were amended in 2012, as a consequence of which regional trauma centers were established by the government to reduce preventable deaths in South Korea. In 2010, when discussions about establishing these centers were in full swing, the proportion of pre- ventable deaths in South Korea was 35\%, which was more than twice that of other advanced countries in the field of trauma [1]. Trauma centers were established in each province, so that patients could reach a trauma center anywhere in the country within an hour, and the trauma center models of advanced countries such as the United States and United Kingdom were benchmarked [2]. 
In today's increasingly subdivided medical system, a multidisciplinary approach is essential to treat severe trauma patients with multiple injuries. However, dedicated specialists from only seven departments participate in the present trauma system: emergency medicine, cardiothoracic surgery, general surgery, orthopedic surgery, neurosurgery, radiology, and anesthesiology. The role of plastic surgeons in treating trauma patients was overlooked when this legal framework was established. In fact, in the field of emergency medicine, plastic surgeons specialize in the simultaneous surgical care of patients with facial trauma, burns, and complicated wounds [1].

Regardless of the increasing awareness of the role of plastic surgeons at trauma centers, their workload has not been investigated in a quantitative manner. The objective of this study was to evaluate the role of the plastic surgery department in treating severe trauma patients.

\section{METHODS}

Following institutional guidelines, this study was approved by the Institutional Review Board of Dankook University Hospital (IRB No. 2020-02-014).

Since 2012 when the law was revised, regional trauma centers have been established every year, and today there are 17 centers nationwide in South Korea. Our hospital has been designated as a regional trauma center from 2012 and the center has officially operated since November 2014. We have run an activation team since January 2012, first as part of preparation for implementation of the center and then as part of its official operations. Thus, this retrospective study was conducted using 7 years of data (from January 2012 to December 2018).

The activation team was deployed when severe trauma patients presented to the emergency room. In general, physiological and anatomical considerations, as well as the mechanism of the injury, were used to determine whether a trauma patient was severe [1]. The Injury Severity Score (ISS) was also used to assess the severity of patients' trauma. The ISS ranges from 0 to 75 points, and severe trauma is usually defined as an ISS greater than 15 points. However, it should be kept in mind that the decision to deploy the activation team is made before a patient is assessed, so the criteria for deploying the activation team may not precisely match the definition of severe trauma patients using retrospective measurements of the ISS.

The activation team was deployed for 14,912 of the 186,247 patients who visited the emergency room during the study period. Of these patients, 2,332 were treated by specialists from the plastic surgery department and 366 patients had an ISS over 15. We conducted a retrospective chart review of those 366 patients
Fig. 1. Severe trauma patients visiting the emergency room

A: Total number of patients who visited the emergency room. B: Number of severe trauma patients who visited the emergency room (B/A: 8.0\%). C: Number of severe trauma patients who were treated by specialists in the plastic surgery department (C/B: 15.6\%). D: Number of severe trauma patients with an Injury Severity Score more than 15 (D/C: 15.7\%).

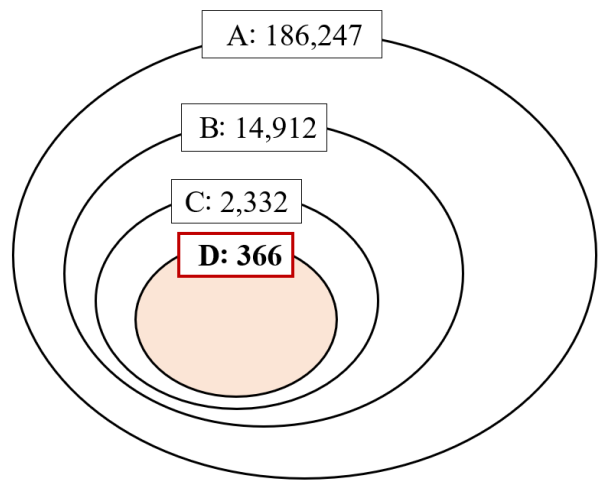

(Fig. 1). Epidemiological data were collected from patients' medical records, including age, sex, ISS, duration of hospitalization (total and in the intensive care unit [ICU]), final outcome, cause of trauma, diagnosis, and departments that collaborated in providing treatment.

\section{RESULTS}

Among the 366 enrolled patients, male patients predominated (298 males [81.4\%] vs. 68 females [18.6\%]). The mean age of all patients was 51.35 years (range, 6-91 years). The average ISS was 22.01 points (range, $16-75$ points). All patients were admitted to the hospital as severe trauma patients, with a mean length of stay of 36.90 days (range, 1-371 days). Among them, 271 patients $(74.0 \%)$ were admitted to the ICU, with a mean length of stay in the ICU of 8.01 days (range, 1-74 days). The most common cause of trauma was traffic accidents (228 patients, $62.3 \%$ ), followed by falls (72 patients, 19.7\%), and occupational injuries (40 patients, 10.9\%). After treatment was completed, most patients were discharged home (227 patients, $62.0 \%$ ), and 14 patients (3.8\%) died (Table 1).

We classified patients' diagnoses based on the anatomical location of trauma and subcategorized injuries as fractures and tissue injuries. The severe trauma patients usually had multiple injuries, and 2.02 plastic surgery diagnoses were made per patient on average (741 diagnoses in 366 patients). Facial trauma was present in the overwhelming majority of cases (705 diagnoses, 95.1\%), and facial bone fractures were the most common type of injury within this category (488 diagnoses, 65.9\%). In detail, 
Table 1. Demographic and clinical data of severe trauma patients treated by plastic surgeons

\begin{tabular}{|lc|}
\hline Variable & Value \\
\hline Sex & \\
Male & $298(81.4)$ \\
Female & $68(18.6)$ \\
Mean age (yr) & $51.35 \pm 18.23$ \\
Mean ISS (score) & $22.01 \pm 6.59$ \\
Mean admission date (day) & \\
General & $36.90 \pm 46.43$ \\
ICU & $8.01 \pm 12.10$ \\
Final outcome & \\
Discharge home & $227(62.0)$ \\
Convalescent hospital & $70(19.1)$ \\
Transfer to area of residence & $53(14.5)$ \\
Death & $14(3.8)$ \\
Discharge AMA & $2(0.5)$ \\
Cause of trauma & \\
Traffic accident & $228(62.3)$ \\
Fall & $72(19.7)$ \\
Occupational & $40(10.9)$ \\
Assault & $9(2.5)$ \\
Slip & $6(1.6)$ \\
Suicide attempt & $2(0.5)$ \\
Natural disaster & $1(0.2)$ \\
Unknown & $8(2.2)$ \\
\hline Values are presented as number (\%) or mean \pm SD. \\
ISS, Injury Severity Score; ICU, intensive care unit; AMA, against medical advice. \\
\hline
\end{tabular}

orbital fractures were the most common type of facial bone fracture (146 diagnoses, 19.7\%), followed by zygomatic fractures (105 diagnoses, 14.2\%) and nasal bone fractures (86 diagnoses, $11.6 \%)$. Meanwhile, facial lacerations were the most common type of tissue injury (207 diagnoses, 27.9\%), followed by burns (30 diagnoses, $4.0 \%$ ) (Table 2).

The departments that collaborated with the plastic surgery department were analyzed because a multidisciplinary approach is an important concept in the treatment of severe trauma patients. Collaboration was done with 1.8 departments on average (663 cases of collaboration among 366 patients). The neurosurgery department accounted for the highest proportion of collaborations ( 245 cases, $37.0 \%$ ), followed by the orthopedic surgery (199 cases, $30.0 \%$ ) and cardiothoracic surgery (150 cases, 22.6\%) departments, respectively (Table 3).

\section{DISCUSSION}

Trauma is a leading cause of death worldwide, especially in reproductive-age individuals; therefore, many countries have established a system for treating trauma patients. According to a reputable report, about 5.8 million deaths occurred by trauma in 2012, and the number of trauma-induced fatalities is expected
Table 2. Diagnoses of the 366 severe trauma patients treated by plastic surgeons

\begin{tabular}{|lllc|}
\hline Location & Character & Diagnosis & No. (\%) \\
\hline Face & Fracture & Orbit & $146(19.7)$ \\
& & Zygoma & $105(14.2)$ \\
& & Nasal & $86(11.6)$ \\
& & Frontal & $42(5.7)$ \\
& Maxilla & $28(3.8)$ \\
& LeFort I & $21(2.8)$ \\
& LeFort II & $17(2.3)$ \\
& Mandible & $16(2.2)$ \\
& LeFort III & $8(1.0)$ \\
& & Panfacial & $8(1.0)$ \\
& & Palate & $6(0.8)$ \\
& & Nasoorbitoethmoidal & $5(0.7)$ \\
& & Laceration & $207(27.9)$ \\
& & Soft tissue defect & $5(0.7)$ \\
Hand & Tissue injury & Lacrimal duct division & $5(0.7)$ \\
& & Phalanx & $2(0.3)$ \\
& & Metacarpal & $2(0.3)$ \\
Body & Tissue injury & Soft tissue defect & $2(0.3)$ \\
Total & Tissue injury & Burn & $30(4.0)$ \\
& & & $741(100.0)$ \\
\hline
\end{tabular}

Table 3. Collaborating departments and number of cases

\begin{tabular}{|lc|}
\hline Department of referral & No. (\%) \\
\hline Neurosurgery & $245(37.0)$ \\
Orthopedic surgery & $199(30.0)$ \\
Cardiothoracic surgery & $150(22.6)$ \\
General surgery & $55(8.3)$ \\
Otorhinolaryngology & $5(0.8)$ \\
Urology & $4(0.6)$ \\
Ophthalmology & $3(0.4)$ \\
Emergency medicine & $2(0.3)$ \\
Total & $663(100.0)$ \\
\hline
\end{tabular}

to increase gradually [3]. In the United States, the foundation of today's regional trauma centers was established by updating guidelines based on the initial guidance from the American College of Surgeons in 1976. This development process has improved trauma patients' outcomes and influenced other countries' trauma systems [4]. In another advanced country in terms of trauma treatment, the United Kingdom, the overall trauma mortality decreased significantly after the introduction of major trauma centers [5]. In contrast, the proportion of preventable deaths in South Korea was 35\% in 2010, which was more than twice that of other advanced countries in the field of trauma [1]. Furthermore, the number of trauma patients has steadily increased, and the mortality of trauma patients rose from $1.87 \%$ in 2007 to $2.71 \%$ in 2011 [3]. In 2012, the South Korean government established a master plan for regional trauma centers, which benchmarked the plans of the United States and the 


\section{United Kingdom [2].}

In the modern medical system, in which clinical specialties are diverse and subdivided, a multidisciplinary approach is essential for the treatment of trauma patients. However, the role of plastic surgeons in trauma has been overlooked. Despite the growing awareness of their role in the emergency room, plastic surgeons' workload in major trauma cases has not been quantified in South Korea. Therefore, obtaining additional insights into the epidemiology of trauma is helpful for establishing treatment strategies and preventing trauma patients, as well as for clarifying the role of plastic surgeons at trauma centers.

Plastic surgeons play a major role in managing trauma patients. Because traumatic damage usually involves soft tissue injuries of the body, facial bone fractures, hand injuries, and neurovascular injuries, plastic surgeons play a highly important role in the treatment of these patients $[1,6]$. For example, after the terrible bomb attack in the 2013 Boston Marathon, plastic surgeons played a substantial role in treating the victims [7]. In our previous study, we statistically demonstrated the importance of plastic surgeons' role in managing trauma patients. Among all emergency room patients, approximately $5 \%$ of patients were treated by plastic surgeons, while the corresponding proportion among trauma patients was roughly $15 \%$ (three times higher) [1]. This result is similar to that of a recent study in the United Kingdom, which found that plastic surgeons treated about $14 \%$ of major trauma patients [8].

In a detailed examination of the epidemiological characteristics of these trauma patients, it was found that face-related injuries $(95.1 \%)$ were the most common diagnosis. Within this category, facial bone fractures were more than twice as common as tissue injuries ( $65.9 \%$ vs. $29.3 \%)$, because the mechanism of severe trauma involves massive force being applied to the patient. In our previous study, the most common types of facial bone fractures caused by traffic accidents were nasal bone fractures (43.6\%), followed by zygomatic (21.3\%) and orbital (20.6\%) fractures. However, orbital fractures (19.7\%) were most common in this study, followed by zygomatic (14.2\%) and nasal bone fractures (11.6\%). Although traffic accidents are high-energy events, previous research only analyzed patients with single facial bone fractures who did not receive collaborative treatment with other departments, and excluded patients who died [9]. Therefore, this study of higher severity patients showed that orbital fractures, which are likely to be caused by high-energy injuries, were more common than other types of facial bone fractures.

Furthermore, the high prevalence of facial bone fractures in severe trauma patients is linked to the finding that collaboration with the neurosurgery department (37.0\%) was more common than collaboration with other departments. Interestingly, in a study of all trauma patients surveyed at another regional trauma center, the craniocerebral area (27.5\%) was the second most frequent trauma area according to results of the Abbreviated Injury Scale (AIS) [3]. In another study, head and neck injuries were only AIS-related factor that was significantly associated with death. The AIS is an anatomical-based coding system that classifies each injury in regions of the body according to its severity [10]. Such results underscore the importance of the role of plastic surgeons in treating damage to the head and neck in trauma situations.

The ISS is a severity index calculated on the basis of the AIS. Severe trauma patients are usually classified as those with an ISS over 15 points [10]. We analyzed severe trauma patients defined according to this criterion, and found a strong male predominance $(81.4 \%)$. In other studies, traffic accidents and occupational injuries, including falls, occurred more often in men than in women. Regardless of developments in medical technology, the expansion of leisure activities and faster transportation have increased the number of trauma cases [3]. In this study, traffic accidents (62.3\%) were the most common cause of injuries, followed by falls (19.7\%) and occupational injuries (10.9\%). In contrast, in the study of Jung et al. [2], a 2-year analysis (from 2015 to 2016) showed that traffic accidents accounted for $43.3 \%$ and falls for $44.5 \%$ of patients with an ISS more than 9. In the United States, the corresponding proportions were 39.5\% and 51.4\%, respectively, during a similar period (from 2013 to 2014). This finding is somewhat different from that of the present study, and it can be seen that the causes of trauma may vary depending on regional characteristics, including socioeconomic, cultural, and environmental factors [11].

Our results presented the overall workload of plastic surgeons at a regional trauma center, and the data contained herein might help in the development of trauma prevention strategies and future workforce planning. However, this study has several limitations. First, this study was retrospective in nature and was carried out at a single trauma center, making it difficult to consider the study fully representative of South Korea. Second, we did not consider other factors that may have influenced patients' outcomes such as their past medical history and family history, because these personal data are difficult to obtain for severe trauma patients in the initial evaluation. Third, the distribution of clinical responsibilities may vary depending on the hospital. For example, in our hospital, hand trauma triage is divided between the plastic surgery and orthopedic surgery departments, and mandibular fractures are divided between the plastic surgery and oral surgery departments.

In conclusion, we scoped out the specialty of plastic surgery 
and its role in the multidisciplinary team of regional trauma centers in this study. These results may have implications for trauma system planning, and we look forward to conducting further investigations to find additional trauma prevention strategies.

\section{NOTES}

\section{Conflict of interest}

No potential conflict of interest relevant to this article was reported.

\section{Ethical approval}

The study was approved by the Institutional Review Board of Dankook University Hospital (IRB No. 2020-02-014) and performed in accordance with the principles of the Declaration of Helsinki.

\section{Author contribution}

Conceptualization: NK Lim. Data curation: JS Jung, NK Lim. Formal analysis: NK Lim. Methodology: JS Jung, NK Lim. Project administration: NK Lim. Visualization: NK Lim. Writing - original draft: JS Jung, NK Lim. Writing - review \& editing: DH Kang, NK Lim.

\section{ORCID}

Joo Sung Jung https://orcid.org/0000-0002-2515-9275

Dong Hee Kang https://orcid.org/0000-0002-6673-9655

Nam Kyu Lim https://orcid.org/0000-0002-0964-983X

\section{REFERENCES}

1. Lim NK, Kang DH. Plan for plastic surgeons to participate in trauma teams at regional trauma and emergency centers. J Korean Med Assoc 2018;61:710-4.
2. Jung K, Matsumoto S, Smith A, et al. Comparison of outcomes in severely injured patients between a South Korean trauma center and matched patients treated in the United States. Surgery 2018;164:482-8.

3. Byun CS, Park IH, Oh JH, et al. Epidemiology of trauma patients and analysis of 268 mortality cases: trends of a single center in Korea. Yonsei Med J 2015;56:220-6.

4. Drolet BC, Tandon VJ, Ha AY, et al. Unnecessary emergency transfers for evaluation by a plastic surgeon: a burden to patients and the health care system. Plast Reconstr Surg 2016; 137:1927-33.

5. Hendrickson SA, Khan MA, Verjee LS, et al. Plastic surgical operative workload in major trauma patients following establishment of the major trauma network in England: a retrospective cohort study. J Plast Reconstr Aesthet Surg 2016; 69:881-7.

6. Igde M, Kaplan A. Plastic surgery management of victims of terrorist violence in Ankara, Turkey. Ann Plast Surg 2017;79: 600-5.

7. Kim PS, Malin E, Kirkham JC, et al. The Boston marathon bombings: the early plastic surgery experience of one Boston hospital. Plast Reconstr Surg 2013;132:1351-63.

8. Hendrickson SA, Staruch RMT, Young K, et al. Major trauma workload and training among UK plastic surgeons: a survey of BAPRAS members. J Plast Reconstr Aesthet Surg 2018;71:1146-52.

9. Choi SH, Gu JH, Kang DH. Analysis of traffic accident-related facial trauma. J Craniofac Surg 2016;27:1682-85.

10. Sim J, Lee J, Lee JC, et al. Risk factors for mortality of severe trauma based on 3 years' data at a single Korean institution. Ann Surg Treat Res 2015;89:215-9.

11. Cabalag MS, Wasiak J, Andrew NE, et al. Epidemiology and management of maxillofacial fractures in an Australian trauma centre. J Plast Reconstr Aesthet Surg 2014;67:183-9. 